Per pendi cul ar and tangent i al angul ar l y resol ved mil ti-si ght neut ral parti cle anal yzer syst em i n LHD

\begin{tabular}{|l|l|}
\hline $\begin{array}{l}\text { jour nal or } \\
\text { publ i cat i on } \mathrm{titl} \text { e }\end{array}$ & Revi ew of Sci ent i f i c I nst r ument s \\
\hline vol une & Vol . 83 \\
\hline number & I ssue10 \\
\hline page range & 10D920-1 - 10D920-3 \\
\hline year & 2012-10-01 \\
\hline URL & ht t p: //hdl . handl e. net /10655/10139 \\
\hline
\end{tabular}




\section{AIP Rersent Scientific Instruments}

Perpendicular and tangential angularly resolved multi-sight neutral particle analyzer system in LHD

T. Ozaki, E. Veshchev, T. Ido, A. Shimizu, P. Goncharov et al.

Citation: Rev. Sci. Instrum. 83, 10D920 (2012); doi: 10.1063/1.4742925

View online: http://dx.doi.org/10.1063/1.4742925

View Table of Contents: http://rsi.aip.org/resource/1/RSINAK/v83/i10

Published by the American Institute of Physics.

\section{Related Articles}

Invited Article: Relation between electric and magnetic field structures and their proton-beam images Rev. Sci. Instrum. 83, 101301 (2012)

Enhanced NIF neutron activation diagnostics

Rev. Sci. Instrum. 83, 10D315 (2012)

Development of a diagnostic technique based on Cherenkov effect for measurements of fast electrons in fusion devices

Rev. Sci. Instrum. 83, 083505 (2012)

Neutron activation diagnostics at the National Ignition Facility (invited)

Rev. Sci. Instrum. 83, 10D313 (2012)

Calibration of neutron-yield diagnostics in attenuating and scattering environments

Rev. Sci. Instrum. 83, 10D914 (2012)

\section{Additional information on Rev. Sci. Instrum.}

Journal Homepage: http://rsi.aip.org

Journal Information: http://rsi.aip.org/about/about_the_journal

Top downloads: http://rsi.aip.org/features/most_downloaded

Information for Authors: http://rsi.aip.org/authors

\section{ADVERTISEMENT}

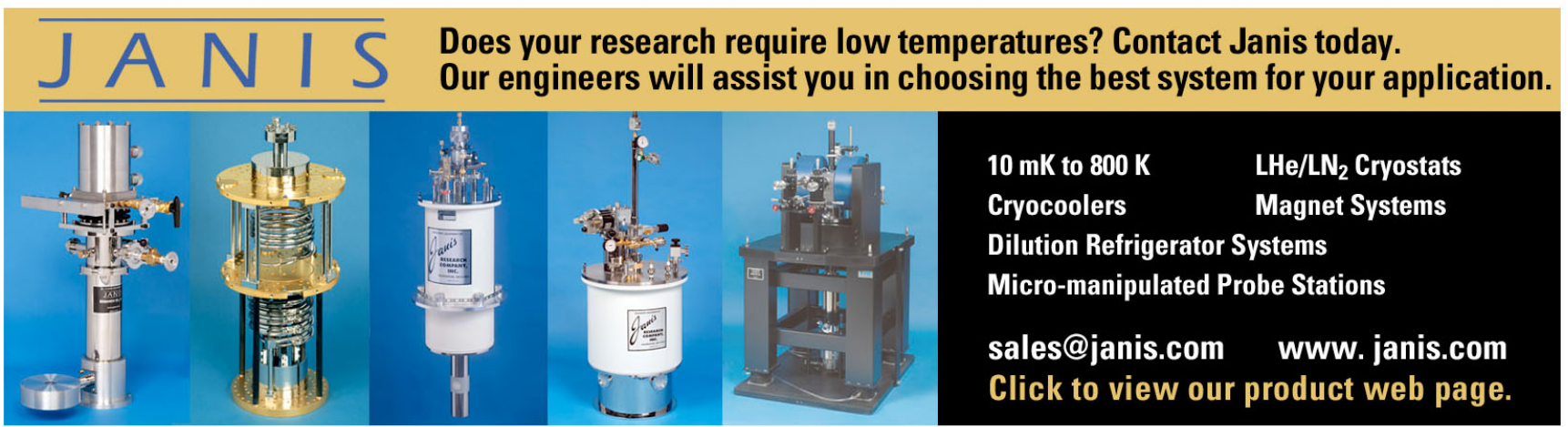




\title{
Perpendicular and tangential angularly resolved multi-sight neutral particle analyzer system in LHD')
}

\author{
T. Ozaki, ${ }^{1, b)}$ E. Veshchev, ${ }^{1,2}$ T. Ido, ${ }^{1}$ A. Shimizu, ${ }^{1}$ P. Goncharov,,${ }^{1,3}$ and S. Sudo ${ }^{1}$ \\ ${ }^{1}$ National Institute for Fusion Science, 322-6, Oroshi, Toki, Gifu 509-5292, Japan \\ ${ }^{2}$ ITER Organization, Cadarache, France \\ ${ }^{3}$ Department of Plasma Physics, Faculty of Physics and Nanotechnology, Saint Petersburg State Polytechnic \\ University, 29 Polytechnicheskaya Street, St. Petersburg 195251, Russia
}

(Presented 9 May 2012; received 6 May 2012; accepted 22 July 2012; published online 24

September 2012)

\begin{abstract}
The particle loss in a helical plasma can be found by measuring the angular distribution of the energetic neutral particles using the angularly resolved multi-sightline neutral particle analyzer (ARMS). In ARMS, the AXUV detector with 20 segments usually used as UV monitor is utilized as the particle detector. Two ARMSs, which are installed with perpendicular and tangential views are operated for measuring the real time neutral particle distribution and investigating the particle loss. (C) 2012 American Institute of Physics. [http://dx.doi.org/10.1063/1.4742925]
\end{abstract}

\section{INTRODUCTION}

Some advantages of a helical device (over a tokamak) include greater plasma stability, longer discharge availability, and wider operating range. However, it has loss mechanism (an orbit loss and a diffusion loss), by which some particles escape from the device. In the Large Helical Device (LHD), ${ }^{1}$ some improvements, for example, tangential neutral beam injection, inner magnetic axis shift, and application of a radial electric field by electron cyclotron resonance heating $(\mathrm{ECH})$, have been tried in order to minimize the particle loss. According to the simulation, ${ }^{2}$ a fraction of the energetic particles cannot be confined in a helical plasma because the orbits of the energetic particle are very complicated. However, the experimental verification has not been performed sufficiently. In order to investigate the particle loss behavior and the energetic particle confinement, the conventional neutral particle analyzer (NPA), the escaped particle detector, ${ }^{3}$ the collective Thomson scattering, ${ }^{4}$ and FICXS (Ref. 5) for measuring the energetic particle spectra have been developed. The NPA with the static electric/magnetic fields is a huge device with poor energy resolution although it has high time resolution as a fundamental diagnostic tool in magnetic fusion devices. In a NPA, a scanning system is required in order to obtain the spatial distribution. To measure the particle loss, the spatial distribution is essential rather than the time resolution. We have been developing an angularly resolved multi-sight line neutral particle analyzer (ARMS-NPA). It has a commercial based xray detector, which enables it to construct inexpensive multimeasuring systems, a compact electrical circuit for signal amplification, which is important for size reduction. Therefore it has compactness, high spatial resolution, suitable time resolution, high-energy resolution, and wide energy ranges.

An energetic neutral particle is produced from an energetic ion from neutral beam injection (NBI) or cyclotron res-

\footnotetext{
${ }^{a)}$ Contributed paper, published as part of the Proceedings of the 19th Topical Conference on High-Temperature Plasma Diagnostics, Monterey, California, May 2012.

b)E-mail: ozaki@nifs.ac.jp.
}

onance heating (ICH) via charge exchange with a background neutral. The energetic particle, which is now a neutral particle, is emitted from the plasma. The background neutral source is mainly from the wall, sometimes fuel/impurity pellet and NBI. Its velocity carries information about the spatial distribution of energetic particles within the plasma because particle loss is strongly dependent on the pitch angle between the local magnetic field line and the velocity vector of the energetic particle. The energetic neutral flux can be assumed to be proportional to the remaining energetic ions. Therefore, the particle loss of the energetic particle can be estimated by measuring the angular and energy distributions of energetic neutral particles. The problem is that the charge exchange measurement is line-integrated. The background neutral profile from the wall is exponentially decreased by interaction with the plasma. However, some background neutrals exist even in the central region (near the magnetic axis) due to the chain reaction of the charge exchange. The energetic ions also have spatial distribution. Therefore, the observed neutral particle origin is further out than the plasma center. Although precise understanding of the ion distribution in a plasma requires all atomic processes and beam orbiting analysis in a simulation, ${ }^{6}$ here we concentrate on understanding the particle orbit qualitatively by introducing the compact neutral particle distribution measurement.

\section{EXPERIMENTAL DEVICE}

ARMS-NPA is based on a PSD (position sensitive detector) in Ref. 7. AXUV (20-segments) as the detector is a commercial product and is reliable as the ultra-violet or soft$\mathrm{x}$-ray detector in current mode. In principle, the response to particles (hydrogen atoms) is similar to that for soft-x-rays because it is a semi-conductor detector. Therefore, proper energy resolution can be expected if the particle flux is limited and the bias is adjusted in the counting mode. Hard x-rays are not strong in helical plasmas except from ECH plasmas. Ultra-violet, soft-x-ray, and visible light can be blocked by a 


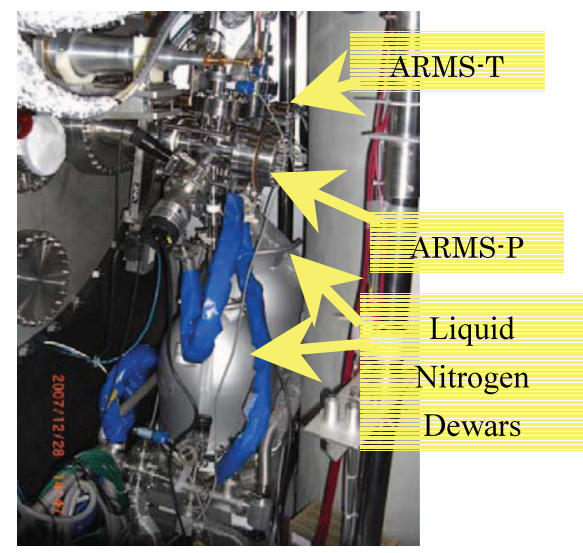

FIG. 1. Photograph of ARMS. Two ARMSs with liquid nitrogen dewars are installed.

thin aluminum film of $0.1-\mu \mathrm{m}$ thickness. The energy loss in Al film is considered by the calculation.

The preamplifier is attached directly to the detector. The minimum observable particle energy of $15 \mathrm{keV}$ is determined by the electrical noise level. The detector and preamplifier are cooled by liquid nitrogen to reduce the electrical noise (see Fig. 1). ARMS has been installed after the calibration by using an Americium 241 gamma-ray source. The size of ARMS is only $15 \mathrm{~cm}$-diameter and $30 \mathrm{~cm}$ length by using the combination with the compact pre-amplifiers circuit and the small detector. ARMS has a time resolution of $5 \mathrm{~ms}$ (1024 time frames), an energy resolution of several keV (2000 energy channels), and a large dynamic range for the particle flux and the flexible viewing angle. The flux is limited by the piezo slit in order to avoid the saturation of the signal and to obtain a wide dynamic range. Two ARMSs, which are installed with perpendicular and tangential views on the 9-O port on LHD, are operated at the same time. Those observable pitch angles on the magnetic axis in LHD are $70^{\circ}-100^{\circ}$ (perpendicular) and $90^{\circ}-140^{\circ}$ (tangential), respectively. Perpendicular ARMS can also measure the vertical or 2-dimentional profile of the neutral flux by using a rotary mechanism. The data are acquired by CAMAC-PHA (Clear Pulse Co.) and WE-7000 (Yokogawa Co.), and are immediately analyzed just after the discharge. In the long discharge on the LHD, there is a capability to obtain the real time, energy/time resolved pitch angle distributions during discharge.

The experiments have been performed at ECH, five NBIs, and the ICH plasmas. Tangential NBI 1 and 3 are injected normally to tangential ARMS but tangential NBI 2 is injected to tangential ARMS by the backscattering. (Perpendicular ARMS is the opposite direction) Perpendicular NBI 4 and 5 are completely in the same specification.

The potential (radial electric field can be obtained by negative gradient of the potential) is monitored by a heavy ion beam probe (HIBP). ${ }^{8}$ HIBP can be operated only at weak and positive magnetic field (= co-direction with the plasma current).

\section{TYPICAL EXPERIMENTAL RESULTS}

As a typical perpendicular ARMS data example, Figure 2 shows the difference of the particle loss when the

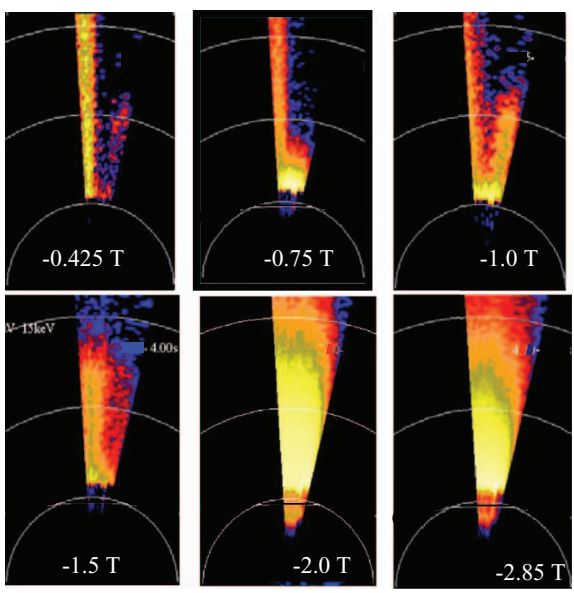

FIG. 2. The particle loss behavior at different magnetic fields. Bright colors indicate increased fluxes. White line shows every $15 \mathrm{keV}$.

magnetic field is changed. In any case, perpendicular NBI 4 has been applied to the tangential NBI plasma. The data are plotted in circular polar coordinates. The radial direction, the declination, and the color show the particle energy, the pitch angle (angle between the magnetic axis and the sightline of ARMS), and the flux in logarithmic scale, respectively. The white lines show the energy ranges of $15 \mathrm{keV}, 30 \mathrm{keV}$, and $45 \mathrm{keV}$. The data are accumulated during 0.25-0.5 s. The magnetic field is written in each figure.

Trapped particles (= high pitch angle component), which come mainly from the perpendicular NBI and from scattered component of the tangential NBI, are well confined even if at the low magnetic field. This is the advantage of the helical device. The particle losses around $80^{\circ}$ are decreased by increasing the absolute value of the magnetic field.

In the passive charge exchange measurement, the signal is line-integrated and has no radial information. Therefore, the comparison between simulation and experiment is necessary. The background neutral and the particle orbits are calculated by the Aurora code and the GNET code, which had been developed by Murakami, respectively. We compare two cases of perpendicular NBI 4 and tangential NBI 1 plasmas measured by the tangential ARMS. At high magnetic field, the neutral particle distribution in the perpendicular NBI 4 plasma is uniformly distributed due to the ion-ion collision in this energy range. The neutral particle distribution obtained from the GNET simulation agrees with that obtained from the experiments. On the tangential NBI 1 plasma, the result qualitatively agrees with the simulation. The tangential component with low pitch angle (transit particles) in the simulation is smaller than that in the experiments. Some of the transit particles escape from the last closed magnetic surface (LCMS) but reenter the plasma again. However in the GNET, the calculation region is limited within the LCMS. The experimental result may show that the re-entering ions cannot be neglected.

The comparison of neutral particle distributions has been performed when the magnetic axis is shifted. In the perpendicular NBI plasma, we can find that the total flux and the uniformity are decreased when the magnetic axis is shifted outward. In the tangential NBI 1 and/or NBI 3 plasmas, the confinement of the transit particles becomes worse. The center 


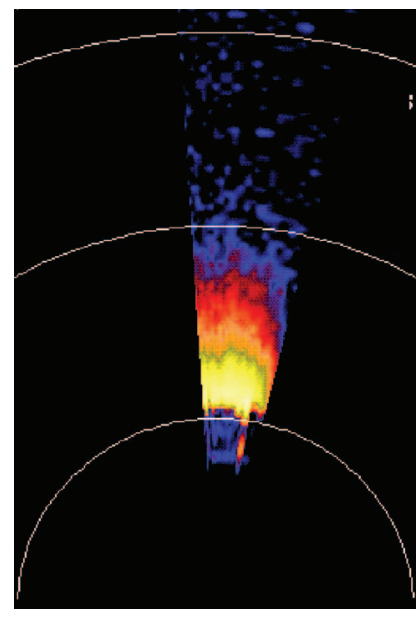

FIG. 3. Perpendicular ARMS during ICH. Rabbit ear structure in the angular distribution can be slightly observed around $70^{\circ}$ of the pitch angle.

of the Poincarè map of the transit particle orbits is about at $\operatorname{Rax}=3.6 \mathrm{~m}$ for any magnetic shifts. Therefore, part of the particle orbit is outside the plasma at the outer magnetic axis.

Figure 3 shows the data during the ICH plasma. The ICH of $38.4 \mathrm{MHz}$ is superposed to the NBI plasma. In ICH plasma, the rabbit-ear structure around the pitch angle of $70^{\circ}$ can be expected because there are many trapped particles at the tip of the banana orbit. Saito et al. measured them by a scanning system of the TOF-NPA shot-by-shot. ${ }^{9,10}$ However, ARMS can observe them in only one discharge.

Figure 4(a) shows the data of the tangential ARMS during the NBI plasma intermittently overlapped by the ECH. Eight frames of every $0.5 \mathrm{~s}$ accumulation are shown just after the discharge. In the second frame, much flux and a uniform distribution can be obtained because only ECH is applied in the low-density plasma. In the fourth frame, the high pitch angle component remains due to the back scattered particle from the NBI 2 (well-confined) when the NBI 1 and NBI 2 are applied. In the fifth frame, the high pitch angle component is recovered when the ECH is overlapped. In the seventh frame, the perpendicular component has almost disappeared when only NBI 3 (badly confined) is applied. In last frame, the high pitch angle component is strongly recovered due to the application of ECH. In this discharge, the potential has been monitored by the HIBP. Figure 4(b) shows the time history of the potential profiles. The symbol colors in Fig. 4(b) correspond to the arrow colors in Fig. 4(a). During ECH application, the electric field (negative potential gradient) in the plasma outer region is positive because the electron escapes from the plasma. The high pitch angle component may be well confined due to the positive electric field. ARMS has a capability of monitoring the real time behavior of the angular distribution of the energetic particle.

\section{SUMMARY}

Perpendicular and tangential ARMS-NPAs have been developed for investigating the particle loss mechanism in LHD. ARMS based on PSD has the spatial resolution of 20 channels (totally 40 channels), $5 \mathrm{~ms}$ time resolution and several $\mathrm{keV}$ energy resolution. ARMS has provided useful data from

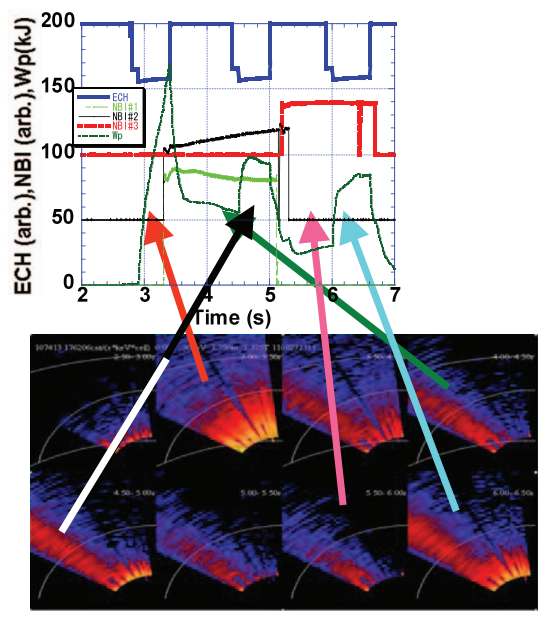

(a)

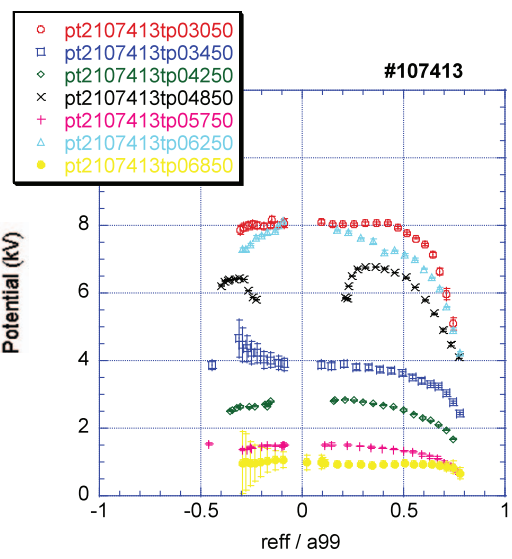

(b)

FIG. 4. (a) Tangential ARMS during NBI+ECH. Angular distribution at every $0.5 \mathrm{~s}$ is shown. Perpendicular particles are recovered by the application of ECH. (b) Plasma potential by HIBP. The last 5 digits in the legend give the timing in milliseconds.

various plasmas. The detector is reliable enough because the experimental results correspond to the simulation results qualitatively. The experimental results are compared with the data from the potential measurement by HIBP. ARMS-NPAs can monitor the real time behavior of the angular distribution of the energetic particles.

\section{ACKNOWLEDGMENTS}

Authors thank the High-Energy Particle Group and HIBP Group, LHD group members, and Dr. T. Watanabe of NIFS, and Dr. S. Murakami of Kyoto University for the support of the simulation. This work is the support of NIFS10, grants-inaid for scientific research.

${ }^{1}$ O. Motojima et al., Fusion Eng. Des. 20, 3-14 (1993).

${ }^{2}$ S. Murakami et al., JPFR Series 5, 620 (2002).

${ }^{3}$ M. Nishiura et al., Rev. Sci. Instrum. 81, 10D313 (2010).

${ }^{4}$ S. Kubo et al., Plasma Fusion Res. 5, S1038 (2010).

${ }^{5}$ M. Osakabe et al., Rev. Sci. Instrum. 79, 10E519 (2008).

${ }^{6} \mathrm{~T}$. Ozaki et al., JPFR Series 7, 22-26 (2006).

${ }^{7}$ E. Veshchev et al., Rev. Sci. Instrum. 77, 10F129 (2006).

${ }^{8}$ T. Ido et al., Rev. Sci. Instrum. 77, 10F523 (2006).

${ }^{9}$ T. Ozaki et al., Rev. Sci. Instrum. 71, 2698 (2000).

${ }^{10}$ K. Saito et al., Plasma Phys. Controlled Fusion 44, 103 (2002). 